\title{
Le droit à un équilibre entre vie professionnelle et vie privée en Espagne
}

\section{Mercedes López Balaguer et Emma Rodríguez Rodríguez}

\section{(2) OpenEdition}

1 Journals

\section{Édition électronique}

URL : https://journals.openedition.org/rdctss/1107

DOI : $10.4000 /$ rdctss. 1107

ISSN : 2262-9815

Éditeur

Centre de droit comparé du travail et de la sécurité sociale

\section{Édition imprimée}

Date de publication : 1 juin 2020

Pagination : 86-93

ISSN : 2117-4350

\section{Référence électronique}

Mercedes López Balaguer et Emma Rodríguez Rodríguez, « Le droit à un équilibre entre vie professionnelle et vie privée en Espagne », Revue de droit comparé du travail et de la sécurité sociale [En ligne], 2 | 2020, mis en ligne le 01 novembre 2021, consulté le 11 novembre 2021. URL : http:// journals.openedition.org/rdctss/1107; DOI : https://doi.org/10.4000/rdctss.1107

\section{cc)}

Revue de droit comparé du travail et de la sécurité sociale est mise à disposition selon les termes de la Licence Creative Commons Attribution - Pas d'Utilisation Commerciale - Pas de Modification 4.0 International. 


\title{
LE DROIT À UN ÉQUILIBRE ENTRE VIE PROFESSIONNELLE ET VIE PRIVÉE EN ESPAGNE ${ }^{1}$
}

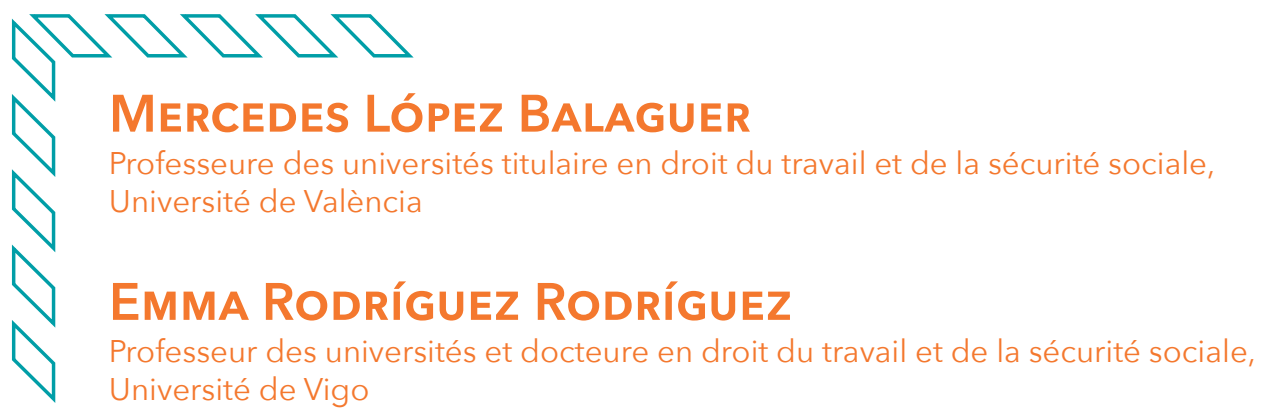

\section{RÉSUMÉ}

Cet article vise à étudier les nouvelles fonctionnalités incorporées à l'art. 34,8ET après la réforme opérée par RDL 6/2019. Ce RDL a considérablement modifié la portée du droit des travailleurs à adapter les horaires de travail pour compatibiliser travail et famille. C'est un droit dont l'exercice génère une importante controverse dans les entreprises comme en témoigne le nombre de jugements en la matière. Par conséquent, l'analyse sera menée dans une perspective très pratique pour évaluer la portée de la réforme de 2019 à travers l'interprétation que les tribunaux font du droit d'adaptation.

Mots-clés : Égalité, coresponsabilité, adaptation de la journée de travail, conciliation

\begin{abstract}
This article aims to study the new features incorporated into art. $34.8 \mathrm{ET}$ after the reform operated by RDL 6/2019. This RDL has significantly modified the scope of the right of workers to adapt working hours to compatibilize work and family. It is a right whose exercise is generating an important controversy in companies as prove the number of judgment on the matter. Therefore, the analysis will be conducted from a very practical perspective to assess the scope of the 2019 reform through the interpretation that the Courts make of the right of adaptation.
\end{abstract}

Key words: Equality, Co-responsibility, Adaptation of Working Day, Conciliation

1 Ce travail s'inscrit dans le cadre des projets de recherche DER2017-83488-C4-3-R « Les droits fondamentaux du travail salarié à l'ère du numérique », et DER2017-83488-C4-2-R "Les droits fondamentaux face à l'évolution du travail indépendant à l'ère du numérique " (ministère des sciences, de l'innovation et de l'enseignement supérieur). 
a question de l'équilibre entre vie professionnelle et vie privée a occupé le devant de la scène dans l'ensemble des États membres de l'Union européenne, devenant le fer de lance du droit à l'égalité des sexes². Traditionnellement, les congés figurent parmi les principaux mécanismes permettant de concilier travail et vie personnelle.

La flexibilité des horaires a, elle aussi, été de plus en plus invoquée pour ajuster les conditions de travail à la vie personnelle et aux obligations familiales des travailleurs, sans avoir à sacrifier les unes au profit des autres. En outre, les autorités publiques ont été appelées à proposer des services adaptés à la prise en charge des adultes et des mineurs dépendants ${ }^{3}$.

Les congés ont joué un rôle majeur mais se sont avérés inefficaces, tandis que la flexibilité des horaires, et plus généralement les mécanismes permettant d'adapter le temps de travail au temps de " non-travail ${ }^{4}$, répondent mieux à l'objectif d'une véritable conciliation. C'est pourquoi, ces situations doivent être appréhendées à travers le prisme du genre ${ }^{5}$.

En 2012, le législateur espagnol a importé l'idée de la «flexisécurité » portée par les institutions de l'Union Européenne, et a promulgué la loi 3/2012 du 6 juillet 2012 relative aux mesures urgentes de réforme du marché du travail ${ }^{6}$. Celle-ci, comme la loi qui la précédait ${ }^{7}$, a fait de la flexibilité du travail son objectif principal. Son chapitre III s'intitule " mesures visant à accroître la flexibilité interne des entreprises pour pallier la destruction d'emplois ", sachant que le recours à la flexibilité interne, comme outil fondamental de l'employabilité, figure dans l'ensemble des dispositions ${ }^{8}$.

Cette tendance à la «flexibilité » des conditions de travail n'est pas nouvelle dans la législation espagnole.

2 M. Bittman et N. Folbre, Family time. The social organization of care, éd. Routledge, London-New York, 2004, p. 1.

3 E. Rodríguez Rodríguez, Institutions permettant de concilier le travail avec la nécessité de s'occuper des proches, Étude de droit comparé, Bomarzo, 2010.

4 M. E. Casas Baamonde, "Organisation du temps de travail sensible à la dimension du genre: conciliation vie personnelle, vie professionnelle », Documentación Laboral, n¹17, 2019, p. 19.

5 En Espagne, $89 \%$ des aidants familiaux sont des femmes, principalement des épouses et des filles, âgées de 45 à 65 ans, selon les chiffres du Conseil supérieur de la recherche scientifique (CSIC). En Espagne, 85 \% des femmes continuent d'effectuer des travaux domestiques et $95 \%$ s'occupent des enfants (Eurostat).

6 Bulletin Officiel de l'État (ci-après BOE), 7 juillet 2012.

7 Décret royal 3/2012 du 10 février, BOE du 11 février 2012.

8 Par exemple, le travail à temps partiel est qualifié de « mécanisme pertinent dans l'organisation flexible du travail ", de même que les heures supplémentaires sont désormais autorisées, ou encore la négociation collective et la priorité accordée à l'accord d'entreprise, justifiée " pour permettre la gestion flexible des conditions de travail ». 
En effet, le chapitre II de la loi 35/2010 du 17 septembre portant mesures urgentes pour la réforme du marché du travail ${ }^{9}$ prévoyait déjà des " mesures pour favoriser la flexibilité interne négociée dans les entreprises et promouvoir la réduction du temps de travail comme instrument d'ajustement temporaire de l'emploi ». En 2010, les instruments favorisant la flexibilité interne avaient été négociés, alors qu'en 2012 les prérogatives de l'employeur ont été considérablement étendues ${ }^{10}$.

Ce renforcement du pouvoir de décision de l'employeur sur les questions de flexibilité interne a accru la vulnérabilité des travailleurs ayant des responsabilités familiales, qui peinent davantage que les autres salariés à s'adapter à des conditions de travail nouvelles et variables. C'est notamment le cas s'agissant des changements de réglementation ayant trait aux conditions de travail - également dénommées "vicissitudes » du contrat de travail - et au système juridique des négociations collectives ${ }^{11}$.

Comme pour compenser cette situation, l'article 40 alinéa 5 du Statut des Travailleurs énonce que "la stabilité professionnelle peut être accordée en priorité aux travailleurs d'autres catégories, tels que les travailleurs soumis à des obligations familiales (...) par le biais d'une convention collective ou d'un accord conclu durant la période de consultation ». Comme l'a relevé par la doctrine ${ }^{12}$, le terme "obligations » utilisé pour désigner les responsabilités familiales en dit long sur la position du législateur. Par ailleurs, cette possibilité étant offerte dans le cadre d'un « accord conclu durant la période de consultation ", seuls les changements de nature collective sont concernés puisqu'en cas de changements individuels, il n'existe pas de période de consultation. En effet, les représentants des travailleurs et la partie intéressée sont seulement « avertis » de la décision de l'entreprise.

Cette disposition pose également d'importants problèmes de fond. Le législateur semble méconnaître le droit fondamental de conciliation, il serait donc vain de l'invoquer dans le cadre d'une convention collective. La jurisprudence du Tribunal constitutionnel est favorable à la reconnaissance du caractère fondamental du droit de concilier vie professionnelle, vie personnelle et vie familiale ${ }^{13}$. Cependant, le renforcement de la flexibilité interne a conduit de nombreux travailleurs ne pouvant faire face aux changements dans l'entreprise, à quitter leur emploi. Autrement dit, la négation du droit fondamental de concilier vie professionnelle et vie familiale a entraîné la mise au banc des travailleurs ayant des responsabilités familiales.

Le législateur espagnol est intervenu à plusieurs reprises sur le droit d'adapter la journée de travail dans la perspective d'une conciliation vie familiale et vie professionnelle à l'origine de décisions judiciaires aussi bien sur les conditions de fond de ce droit (I) que sur les garanties procédurales (II).

9 BOE du 18 septembre 2010. Ce règlement est dans la lignée de la loi RD 10/2010 du 16 juin (BOE du 17 juin 2010).

10 F. Valdés Dal-Ré, « La flexibilité interne dans la loi 3/2012 », RL, n¹5-18, août 2012: http://revistas. laley.es/Content/Documento

11 ld.

12 M. A. Ballester Pastor, "Comment la réforme opérée par la loi RD 3/2012 a entaché le caractère fondamental du droit de conciliation des responsabilités », RDS, n57, p. 105.

13 STC 3/2007 du 15 janvier 2007. 


\section{I - LE DROIT À L'ADAPTATION DE LA JOURNÉE DE TRAVAIL POUR CONCILIER VIE FAMILIALE ET VIE PROFESSIONNELLE}

Dans le système juridique espagnol, la modification de l'article 2(8) du Décret-loi royal 6/2019 du $1^{\text {er }}$ mars $2019^{14}$ a supposé l'ajustement du droit d'adaptation de la journée de travail prévu par l'article 34.8 du Statut des travailleurs, ce qui répond dans une large mesure aux dispositions de la nouvelle Directive 2019/1158.

Intégré au texte de l'article 34 du Statut des travailleurs par la loi organique 3/2007 du 22 mars 2007 relative à l'égalité effective entre femmes et hommes, cet alinéa 8 s'inscrit dans la lignée d'une série de mesures visant à favoriser l'équilibre entre vie professionnelle et vie familiale. À son tour, la loi 3/2012 du 6 juillet 2012 relative aux mesures urgentes de réforme du marché du travail a révisé le texte de l'article 34.8 du Statut des travailleurs, en y ajoutant un second paragraphe. Or depuis son approbation, la portée du droit à l'adaptation de la journée de travail, jusqu'alors régi par l'article 34.8 du Statut des travailleurs, est remise en question par la jurisprudence ${ }^{15}$. Avec la réglementation antérieure, et compte tenu de l'absence de mesures normalisées applicables en la matière, l'adaptation de la journée de travail pouvait n'aboutir à rien, même si certaines juridictions considéraient que l'entreprise devait apporter des explications objectives pour justifier sa réponse négative, en raison de la nature constitutionnelle du droit et des intérêts en jeu ${ }^{16}$.

Depuis la réforme apportée par le Décret-loi royal 6/2019, l'article 34.8 du Statut des travailleurs régit expressément le droit des salariés à «demander l'adaptation de la durée et de la répartition de leur journée de travail, en matière d'aménagement du temps de travail et de forme de la prestation, y compris le travail à distance, pour rendre effectif leur droit à un équilibre entre vie familiale et vie professionnelle». La référence antérieure à l'équilibre entre vie professionnelle et vie «privée» disparaît donc, même si sa répercussion était imperceptible dans la pratique ${ }^{17}$.

Avec la réforme de 2019, la demande d'adaptation de la journée de travail se limite au besoin des salariés de concilier vie professionnelle et vie de famille. Les limites de la portée subjective de cette adaptation doivent donc être rattachées aux sujets qui se trouvent à l'origine de ce droit. À cet égard, la règle édictée comprend aujourd'hui une référence qui n'existait pas auparavant, à savoir la limite d'âge, lorsque la personne à l'origine de laquelle s'exerce ce droit est un enfant. Cette limite d'âge est fixée à 12 ans. Concrètement,

14 Décret-loi royal 6/2019, du 1er mars 2019 sur les mesures urgentes garantissant l'égalité de traitement et l'égalité des chances entre les hommes et femmes en matière d'emploi et de travail, BOE du 7 mars 2019.

15 Voir par exemple les décisions du Tribunal suprême des 13 et 18 juin 2008, Rec. 897/07 et 1625/07.

16 Décision du Tribunal Superior de Justicia de Asturias du 18 décembre 2009, Rec. 2738/09; Décision du Tribunal Superior de Justicia de Galicia du 20 mai 2010, Rec. 4392/09 ; Décision du Tribunal Superior de Justicia de Andalucía du 5 avril 2017, Rec. 2736/16; Décision du Tribunal Superior de Justicia de Madrid du 28 novembre 2018, Rec. 971/2018.

17 G. Rodríguez Pastor, «Tiempo de trabajo tras la reforma operada por la LOI», in AAVV, Los aspectos laborales de la Ley de Igualdad, Tirant lo Blanch, 2007, p. 80 ; C. Alfonso Mellado, «El tiempo de trabajo en la Ley Orgánica para la igualdad efectiva de mujeres y hombres ", in AAVV, Comentarios a la Ley Orgánica 3/2007, de 22 de marzo, para la igualdad efectiva de mujeres y hombres, Madrid, La Ley, 2008, p. 412. 
le texte dispose que "lorsqu'il/elle a des enfants, le salarié / la salariée peut introduire une demande jusqu'à ce que lesdits enfants atteignent l'âge de 12 ans».

Ainsi, le législateur assortit au droit d'adaptation de la journée de travail la même limite d'âge des enfants que celle assortie aux hypothèses de réduction du temps de travail pour garde d'enfants (article 37.6 du Statut des travailleurs). Dès lors, le droit de demander l'adaptation de la journée de travail pour une conciliation liée aux enfants ne pourra être exercé que durant ce laps de temps, et non après ${ }^{18}$. Bien entendu, cette limite renvoie aux hypothèses supposant une attention ou des soins intrinsèquement liés à la minorité de l'enfant, pouvant nécessiter une attention particulière justifiée par des circonstances objectives.

Outre les enfants - et comme dans la réglementation antérieure - le droit à demander l'adaptation de la journée de travail est également applicable aux cas de prise en charge de membres de la famille. Avec l'absence de toute limite introduite par la loi modifiée concernant les membres de la famille, il conviendra d'opter pour une interprétation au sens large, selon laquelle la demande d'équilibre entre vie familiale/professionnelle ne se limitera pas aux conditions fixées par l'article 37.6 du Statut des travailleurs sur les liens familiaux - personnes jusqu'au deuxième degré de parenté ou d'affinité qui, en raison de leur âge, d'un accident ou de maladie, ne peuvent être autonomes et n'exercent pas d'activité rémunérée -, mais pourra s'étendre au-delà.

Concernant le champ d'application objectif, le droit régi par l'article 34.8 après la réforme de 2019 permet au salarié de présenter à son entreprise une demande portant sur l'adaptation, d'une part de la durée et de la répartition de la journée de travail, d'autre part de l'aménagement du temps de travail, et enfin de la forme de la prestation, y compris le travail à distance. Pour ce qui est de l'adaptation de la durée et de la répartition du temps de travail, la portée de cette disposition ne modifie pas la lettre de la loi, puisqu'elle était déjà reconnue en ces termes. En revanche, les mentions relatives à l'aménagement du temps de travail, à la forme de la prestation du service, ainsi qu'au travail à distance, constituent pour leur part des nouveautés.

De fait, la jurisprudence refusait aux salariés le droit de modifier - sans la réduire leur journée de travail en vertu de l'article 37.6 du Statut des travailleurs, arguant que cette hypothèse n'était pas prévue par le texte de loi et qu'une demande d'adaptation sans réduction de la journée de travail se trouvait dès lors "dépourvue de fondement juridique $»^{19}$. Même avant l'introduction de l'article 34.8 dans le Statut des travailleurs, les juridictions exhortaient le législateur à reconnaître le droit d'adaptation de la journée de travail, étant entendu qu'il "serait recommandé et souhaitable d'introduire une plus grande souplesse sur le lieu de travail englobant, pour la salariée, la possibilité de choisir ses horaires de travail en fonction de sa nouvelle situation personnelle, sans réduire sa journée de travail, afin d'assurer un maximum de compatibilité et d'adaptation entre sa vie

18 Par opposition à cette interprétation, le Juzgado de lo Social $n^{\circ} 1$ de Valladolid a estimé, dans sa décision 146/2019 du 22 novembre 2019, que la limite d'âge de 12 ans ne devait pas entraver l'exercice du droit concerné une fois cet âge dépassé par les enfants.

19 Décision du Tribunal Superior de Justicia de Andalucía du 23 décembre 2008, Rec. 2059/08 ; Décision du Tribunal Superior de Justicia de Cataluña du 7 février 2008, Rec. 7922/06 ; Décision du Tribunal Superior de Justicia de Madrid du 19 novembre 2007, Rec. 3653/08 ; Décision du Tribunal Superior de Justicia de Cataluña du 7 mars 2017, Rec. 7164/2016. 
professionnelle et sa vie personnelle caractérisée par la garde d'un mineur de six ans, en combinant les deux aspects et en éliminant les obstacles existants $»^{20}$.

L'inclusion, dans l'article 34.8 du Statut des travailleurs, de la possibilité de demander la modification de la forme de la prestation du service comme formule d'équilibre entre vie professionnelle et vie familiale est novatrice.

En premier lieu, ce qui attire l'attention c'est précisément le fait que cette référence soit incluse dans l'article de la Section 5 du chapitre II du Statut des travailleurs, qui régit exclusivement la journée de travail, puisque le changement de forme de la prestation peut bien entendu supposer une modification du temps de travail comme condition essentielle de la prestation de travail, mais implique de façon évidente la modification du mode d'exercice de cette prestation et va bien au-delà du seul temps de travail.

Dans tous les cas, la possibilité explicite de demander la réalisation du travail à distance est très intéressante du point de vue de l'équilibre entre vie professionnelle et familiale. Cette possibilité est ainsi prévue à l'article 3.1.f) de la Directive 2019/1158 susmentionnée, qui définit les formules de travail flexible comme "une possibilité pour les travailleurs d'aménager leurs régimes de travail, y compris par le recours au travail à distance, à des horaires de travail souples ou à une réduction du temps de travail ». De fait, la formule du travail à distance comme moyen pour assurer l'équilibre est déjà mise en œuvre dans la pratique dans le cadre de conventions collectives ou bien, s'agissant de l'emploi public, de la réglementation interne ${ }^{21}$.

\section{II - LES GARANTIES RELATIVES À L'EXERCICE DES DROITS À L'ÉQUILIBRE}

La réglementation post-réforme de 2019 semble beaucoup plus claire quand il s'agit de cerner la procédure applicable aux demandes d'adaptation du temps de travail. La reconnaissance d'un véritable droit des salariés à l'adaptation de la journée de travail, en vue de parvenir à l'équilibre entre vie professionnelle et vie familiale, apparaît elle-même beaucoup plus claire.

En effet, les règles de procédure à suivre pour que la demande d'adaptation de la journée de travail ou de la forme de prestation du travail du salarié puisse aboutir permettent d'affirmer que ladite demande, comme la réponse de l'entreprise, devront être ajustées à la nécessaire preuve des causes objectives concurrentes. Ces dernières sont liées à la recherche d'un équilibre pour le salarié, et aux besoins organisationnels et de production pour l'employeur.

Dans la pratique, cette procédure correspond à celle applicable aux cas de réduction de la journée de travail, qui consiste à demander et à répondre aux besoins d'adaptation. En effet, selon l'interprétation des juridictions, le droit du salarié à l'organisation de son emploi du temps, et à la détermination de la période de jouissance prévue à l'article 37.7 du Statut des travailleurs, suppose forcément l'obligation pour ce dernier de

20 Décision du Tribunal Superior de Justicia de la Comunidad Valenciana du 10 mai 2005, Rec. 3226/04.

21 L. Mella Méndez, (dir.), El teletrabajo en España: aspectos teórico-prácticos de interés, La Ley, Madrid, 2016, p. 30. 
prouver que cette nouvelle journée est celle qui s'ajuste le mieux à ses besoins en termes d'équilibre.

En outre, la nouvelle procédure prévue par l'article 34.8 du Statut des travailleurs répond également, d'une part à la procédure de l'article 139 de la loi sur les juridictions sociales - qui impose que les propositions et solutions alternatives émises par les parties soient présentées au juge - et, d'autre part, aux dispositions de l'article 9 de la Directive $2019 / 1158$.

À cet égard, cette dernière réforme de 2019 représente une occasion manquée d'unifier, grâce à la loi, la procédure à suivre pour toute demande de changement du temps de travail visant à exercer le droit à l'équilibre entre vie professionnelle et familiale. Le législateur aurait peut-être dû mener une réforme plus ambitieuse - donc plus efficace - en matière de droits à l'équilibre du point de vue de son application pratique, en fixant systématiquement une procédure unique ad hoc en l'absence de réglementation conventionnelle $e^{22}$.

Si la casuistique est évidemment importante dans ce domaine, le fait est qu'en général, les demandes présentant des incompatibilités en matière de soins familiaux - qu'elles soient liées à l'emploi du temps de l'autre parent, de l'école maternelle, de l'établissement scolaire, du centre de jour, etc. - sont réputées conformes au droit ${ }^{23}$. En effet, il est considéré que le droit à l'équilibre entre vie professionnelle et vie familiale prévaut lorsque l'entreprise ne justifie pas son refus en attestant de l'existence de motifs concurrents de nature organisationnelle ou productive ${ }^{24}$.

De plus, la jurisprudence estime que ne suffisent pas à justifier la demande d'adaptation : - la simple allusion à un besoin général de soins familiaux ${ }^{25}$;

- la volonté des deux parents d'organiser leur journée pour travailler ensemble, en marge de leur droit à l'équilibre entre vie professionnelle et familiale ${ }^{26}$;

- et, concernant l'exercice pratique du droit à l'équilibre en matière de coresponsabilité, la demande présentée " sans que soient attestées les circonstances concrètes de la scolarisation, les besoins du mineur et l'incidence des conditions de travail concrètes des deux parents qu'il convient, du point de vue de la coresponsabilité, en l'espèce en matière de tâches familiales, (conformément aux Directives du Conseil 92/85/CEE du 19 octobre 1992, et 96/34/CE, du 3 juin 1996, modifiée par la Directive 97/75/CE) de considérer comme étant raisonnables ${ }^{27}$.

22 Sur ce processus, voir C. Fernández Prats, E. García Testal, et M. López Balaguer, Los derechos de conciliación en la empresa. Actualizado al RDLey 6/2019, de 1 de marzo, de medidas urgentes para garantía de la igualdad de trato y de oportunidades entre mujeres y hombres en el empleo y en la ocupación, Tirant lo Blanch, Valence, 2019, p. 117.

23 La décision du Tribunal Superior de Justicia de Asturias du 22 janvier 2019, Rec. 2815/2018 est très explicite à ce sujet, puisqu'elle donne une importance particulière aux motifs exposés par la salariée. Voir également la décision du Juzgado de lo Social d'Oviedo du 28 février 2018, Proc. 109/2018.

24 Décision du Tribunal Superior de Justicia de Madrid du 28 septembre 2018, Rec. 345/2018 ; Décision du Tribunal Superior de Justicia de Guadalajara du 7 mars 2018, Proc. 102/2018.

25 Décision du Tribunal Superior de Justicia del País Vasco du 18 décembre 2018, Rec. 2392/2018.

26 Décision du Tribunal Superior de Justicia de Madrid du 20 mars 2018, Rec. 728/2017.

27 Décision du Tribunal Superior de Justicia de Valladolid du 14 mars 2018, Proc. 1057/2017. 
Avec cette nouvelle disposition, le Décret-loi royal 6/2019 susmentionné introduit dans le texte législatif une condition considérée comme essentielle, tant par la doctrine scientifique que par certaines juridictions, du point de vue de la dimension constitutionnelle du droit à l'équilibre tel que reconnu par le Tribunal constitutionnel dans sa décision 3/2007 du 15 mars 2007. Désormais pondérée par l'entreprise qui l'évalue et la négocie, sur la base de critères objectifs, la demande d'adaptation de la journée de travail de tout salarié est ainsi devenue une obligation reconnue par l'article 34.8 du Statut des travailleurs, qui prévoit une procédure s'articulant de préférence au niveau collectif, et à défaut individuel.

En conclusion, la procédure actuelle de demande d'adaptation de la journée de travail, en vue de la recherche d'un équilibre entre vie professionnelle et vie familiale, offre aux salariés une solution plus pratique pour ajuster leurs conditions de travail à leurs besoins en matière d'équilibre. Il est probable qu'à l'avenir, le nombre de demandes de réduction de la journée de travail diminue, et que celui des demandes d'adaptation sans réduction de la journée de travail augmente. Bien que cela dépende évidemment des situations, l'adaptation de la journée de travail pourrait en effet s'avérer dans la pratique la solution la plus adaptée aux salariés en général, pour parvenir à l'équilibre recherché.

En ce qui concerne le droit d'adaptation de la journée de travail, la réforme de 2019 est toutefois critiquable du point de vue de la coresponsabilité, pourtant bien prise en compte par le législateur dans le Décret-loi royal 6/2019 modifiant les droits liés aux congés pour naissance. Par ailleurs, il était nécessaire de prévoir des dispositions législatives régissant l'utilisation alternative du droit d'adaptation des salarié(e)s par rapport aux personnes à l'origine dudit droit - qu'il s'agisse de descendants ou d'autres membres de la famille afin de dépasser le rôle traditionnel d'aidantes endossé par les femmes qui, en définitive, perpétue et accroît les disparités entre les sexes sur le marché du travail.

Nonobstant ces insuffisances, le système d'adaptation actuellement régi par l'article 34.8 du Statut des travailleurs améliore considérablement le précédent et confère une plus grande sécurité juridique, tant aux salariés qu'aux entreprises, en ce qui concerne l'exercice du droit. Les raisons objectives formulées de part et d'autre constitueront l'élément essentiel à prendre en compte au moment de la prise de décision quant à la prévalence de la demande du salarié, ou quant à la nécessité d'ajuster cette demande aux exigences organisationnelles de l'entreprise.

En ce sens, la négociation collective devra constituer le cadre naturel de régulation de ce droit, en supposant que l'autonomie collective serve à concrétiser et à délimiter les conditions de son exercice dans les secteurs ou les entreprises. 